\title{
Superconductivity without dependence on valence electron density in (Al, Zn, Co) doped YBCO systems
}

\author{
Zhang Yufeng \\ Department of Mathematics and Physics, Shanghai University of Electric Power \\ 28 Xuehai Road, Nanhui District, Shanghai 201300, PRC \\ E-mail: zyfeng1011@yahoo.com.cn \\ Wang Dandan and Li Pinglin \\ School of Physics and Engineering, Zhengzhou University, Zhengzhou 450052, PRC
}

Received July 30, 2009, revised September 1, 2009

\begin{abstract}
We adopted the x-ray diffraction, oxygen contents, positron annihilation technology and simulation methods to investigate systematically $\mathrm{YBa}_{2} \mathrm{Cu}_{3-x}(\mathrm{Al}, \mathrm{Zn}, \mathrm{Co})_{x} \mathrm{O}_{7-\delta}(x=0-0.5)$ cuprates. The experimental results and simulated calculations support the existence of cluster effect. Moreover, it is concluded that the cluster effect is an important factor on suppression of the superconductivity and the $T_{c}$ does not depend directly on the density of valence electron in the samples.
\end{abstract}

PACS: 68.65.-k Low-dimensional, mesoscopic, nanoscale and other related systems: structure and nonelectronic properties;

47.54.De Experimental aspects;

47.54.Bd Theoretical aspects.

Keywords: YBCO, simulated calculations, positron annihilation, cluster effect, valence electron.

\section{Introduction}

The high- $T_{c}$ cuprates, especially YBCO systems, are still the highlight in the superconducting researches [1-5], however, the microscopic mechanism is challenging harshly to all theories, even as Zaanens statements: «The high- $T_{c}$ superconductivity is on the list of the most profound physics problems...» [6]. In order to search and comprehend such a problem, scientists suggested many new theories and experimental technologies [7-9], for example, elemental substitution that play an important role in the research of high- $T_{c}$ cuprates until today [10-13], namely, copper is replaced by magnetic or nonmagnetic elements. According to the traditional theory of magnetic pair-breaking, the nonmagnetic ions doped into superconductors will suppress less the superconductivity than magnetic ions. However, $\mathrm{Zn}^{2+}$ doped in YBCO, a kind of nonmagnetic ion, suppresses more forcefully the superconductivity than other nonmagnetic and magnetic ions, like $\mathrm{Al}$ and Co. At present, most researchers believe that the superconducting phenomenon occurs on $\mathrm{CuO}_{2}$ planes [1416], where $\mathrm{Zn}^{2+}$ ions substitute for $\mathrm{Cu}(2)$ sites. Hence, $\mathrm{Zn}$ doping will suppress noticeably the superconductivity $[17,18]$. Some scientists suggested $\mathrm{Zn}$ doping destroys the background of antiferromagnetism on $\mathrm{CuO}_{2}$ planes [19]; however, this proposal is still one of viewpoints of magnetic pair-breaking. Besides, what a reason causes the transition temperature $T_{c}$ to fall faster and faster with the increase of doped concentration [20-25] and so on? Many queries are now still unclear; therefore, further investigations of theories and experiments will be necessary in $\mathrm{Al}$, $\mathrm{Zn}$ and Co doped YBCO cuprates.

In order to realize the distribution of doped ions, here we will calculate the total defect energy and average binding energy inside a cluster based on Islam's method [26]. Simulated results reveal the possibility of cluster effect. When the doped concentration increases, the doped ions combine into different clusters on $\mathrm{CuO}_{2}$ planes and in $\mathrm{CuO}$ chains. Afterwards, we use positron annihilation technique (PAT) to probe the change of electron structure as Al, $\mathrm{Zn}$ and $\mathrm{Co}$ substitute for $\mathrm{Cu}$ in YBCO. PAT plays an important role in the investigation of such condensed matters as semiconductors, metal materials and high- $T_{c}$ cuprates [2730]. The positron studies examine the properties of normal and superconducting states, such as Fermi surface, O-T transition and the carrier concentration [31-34]. In recent years, Jean and Li et al. reported many important results from positron experiments and theories concerning high- $T_{c}$ superconducting mechanism [20-24,33-35]. In the present article, we report the systematical investigation concerning 
$\mathrm{YBa}_{2} \mathrm{Cu}_{3-x}(\mathrm{Al}, \mathrm{Zn}, \mathrm{Co})_{x} \mathrm{O}_{7-\delta}(x=0-0.5)$ samples. The theoretic calculations and experimental results of PAT and oxygen content support the existence of cluster effect. Moreover, it is reasoned that cluster effects, as an important factor, suppress the cuprate superconductivity and the $T_{c}$ variations do not directly depend on the density of valence electron in the samples.

\section{Experiments}

Samples of $\mathrm{YBa}_{2} \mathrm{Cu}_{3-x}(\mathrm{Al}, \mathrm{Zn}, \mathrm{Co})_{x} \mathrm{O}_{7-\delta}(x=0-0.5)$ were prepared by the same method as in Refs. 20 and 22-24. Due to the sensitivity of the PAT experiments, all samples were sintered in the same conditions in order to reduce the dispersion of the experimental results. The superconducting transition temperature $T_{c}$ was measured by the standard dc four-probe method with a voltage resolution of $10^{-7} \mathrm{~V}$ (HP3457A). The crystal structures of samples were analyzed by powder $\mathrm{x}$-ray diffraction (XRD) using the D/max-BX-ray diffractometer. The positron lifetime spectra were measured by the ORTEC-100U fast-fast coincidence lifetime spectrometer. Two pieces of identical samples $(\varnothing 13 \times 3 \mathrm{~mm})$ were sandwiched together with a $10-\mu \mathrm{C} 22 \mathrm{Na}$ positron source deposited on a thin Mylar foil (about $1.2 \mathrm{mg} / \mathrm{cm}^{2}$ thickness). We apply Pilot-U plastic flicker sensor, which was tested by ${ }^{60} \mathrm{Co}$ and showed the excellent time resolution over 220 ps. Each spectrum contains more than $1 \cdot 10^{6}$ counts to guarantee the sufficient statistic precision. After subtracting background and source contributions, the lifetime spectra were fitted with twolifetime components by POSITRON-FIT-EXTENDED program with the best fit $\left(\chi^{2}=1.0-1.1\right)$. The positron lifetime spectra of all samples were measured at the identical environment temperature, $(283 \pm 1) \mathrm{K}$, and the results were reproducible.

\section{Results and discussions}

\subsection{Simulated calculations}

In order to understand the characteristics of cluster structure, here we perform simulated calculations on the energy minimization principle and framework of Born model, in which the effective pairwise potentials represent the interatomic forces in the following form [26]:

$$
\Phi_{i j}=\frac{Z_{i} Z_{j} e^{2}}{4 \pi \varepsilon_{0} r_{i j}}+A_{i j} \exp \left(-\frac{r_{i j}}{\rho_{i j}}\right)-\frac{C_{i j}}{r_{i j}^{6}},
$$

where $\Phi_{i j}$ is the effective potentials between ions $i$ and $j ; Z_{i}$ and $Z_{j}$ are ion valences; $r_{i j}$ is the distance between ions $i$ and $j ; A_{i j}, C_{i j}$ and $\rho_{i j}$ are the relative characteristic constants. The first term is the long-range Coulomb interaction; the remaining terms represent the short-range interaction and the shielded revision. Because charge equilibrium determines the ion coordination characteristics in clusters, the clusters should be electrically neutral. The neutral clusters may show several structures, two of which are illustrated as $(a)$ and $(b)$ on $\mathrm{CuO}_{2}$ planes in Refs. 20-24. Here (a) as Hexamer denotes six doped ions with two commonlateral squares, and $(b)$ as Double Square does seven doped ions with two common-apical squares. $\mathrm{On} \mathrm{CuO}_{2}$ planes $\mathrm{Cu}$ ions are +2.25 valence, and doped $\mathrm{Zn}$ ions are +2 , moreover holes exist on oxygen ions $[25,36]$. These two factors together make electrons lose; consequently, the number of the oxygen ions has to decrease in order to keep the charge equilibrium. For example, in four doped ions cluster, every four $\mathrm{Zn}^{2+}$. ions may exclude an oxygen ion, which can be described as $\left\{4 \mathrm{Zn}^{2+} \rightarrow 4 \mathrm{Cu}^{2.25+}-\mathrm{O}^{2-}\right\}$. Nevertheless, one $\mathrm{Zn}$ ion alone cannot exclude an oxygen ion. Hence, the binding energy of $\mathrm{Zn}^{2+}$ cluster is:

$$
\begin{aligned}
& E_{\text {bind }}=E_{b 1}\left(4 \mathrm{Zn}^{2+} \rightarrow 4 \mathrm{Cu}^{2.25+}-\mathrm{O}^{2-}\right)- \\
& -4 E_{b 2}\left(\mathrm{Zn}^{2+} \rightarrow \mathrm{Cu}^{2.25+}\right)+E_{b 3}\left(\mathrm{O}^{2-}\right) .
\end{aligned}
$$

where $E_{b 1}, E_{b 2}$ and $E_{b 3}$ are all represented as $E=\sum \Phi_{i j}$. The first term $E_{b 1}$ is the cluster total energy, $E_{b 2}$ is the binding energy that a $\mathrm{Zn}^{2+}$ substitutes for a $\mathrm{Cu}^{2.25+}$ ion, and $E_{b 3}$ is the binding energy that an $\mathrm{O}^{2-}$ ion is lost. For both Hexamer and Double Square, $E_{\text {bind }}$ have similar formulation. The average binding energy of every doped ion is $E_{\text {mean }}=E_{\text {bind }} / N$ where $N$ is the number of doped ions in a cluster. Other clusters may likewise be described and calculated. The calculation results are listed in Table 1, in which the negative value of the binding energy indicates the system is bound. As well known, the larger average binding energy is, the more stable a cluster combines. Consequently, the doped ions should prefer to form the cluster with largest average binding energy. It can be concluded from the results listed in Table 1 that doped $\mathrm{Al}$ and Co ions prefer to form Hexamer cluster of six ions, while for $\mathrm{Zn}$ doping Double Square (a common point, seven ions) clusters have the largest probability. As for the larger cluster, it can be composed by some small clusters, for example, the cluster of eight ions are composed by double four ions and so on.

Table 1. The mean binding energy of per doped ion in the doped YBCO

\begin{tabular}{c|c|c|c|c}
\hline \hline Cluster & $\mathrm{Al}^{3+}-E_{m}$ & $\mathrm{Co}^{3+}-E_{m}$ & Cluster & $\mathrm{Zn}^{2+}-E_{m}$ \\
\hline \hline $\mathrm{D}+\mathrm{O}$ & -2.73 & -2.80 & $\mathrm{D}$ & -1.67 \\
$\mathrm{TL}+2 \mathrm{O}$ & -2.25 & -2.44 & $\mathrm{TL}-\mathrm{O}$ & -1.49 \\
$\mathrm{TS}+2 \mathrm{O}$ & -2.71 & -2.82 & $\mathrm{TS}-\mathrm{O}$ & -1.64 \\
$\mathrm{TZ}+2 \mathrm{O}$ & -2.10 & -2.15 & $\mathrm{TZ}-\mathrm{O}$ & -1.36 \\
$\mathrm{H}+3 \mathrm{O}$ & -2.83 & & $\mathrm{H}-2 \mathrm{O}$ & -1.68 \\
$\mathrm{H}+4 \mathrm{O}$ & -2.85 & -3.03 & $\mathrm{H}-\mathrm{O}$ & -1.75 \\
$\mathrm{DS}+4 \mathrm{O}$ & -2.81 & -2.91 & $\mathrm{DS}-2 \mathrm{O}$ & -1.78 \\
\hline \hline
\end{tabular}

$\mathrm{N}$ o t e s: Here the negative value indicates the binding energy. $E_{m}$ (eV/ion) is the mean binding energy per doped ion. $\mathrm{D}+\mathrm{O}=$ Dimmer $\left\{2 \mathrm{M} \rightarrow 2 \mathrm{Cu}^{2+}+\mathrm{O}^{2-}\right\} \quad\left(\mathrm{M}=\mathrm{Al}^{3+}, \mathrm{Co}^{3+}\right), \ll+\mathrm{O} »$ represents an $\mathrm{O}^{2-}$ ion is attracted into the cluster;

$\mathrm{TL}+2 \mathrm{O}=$ Tetramer $\left\{4 \mathrm{M} \rightarrow 4 \mathrm{Cu}^{2+}+2 \mathrm{O}^{2-}\right\}$ Linear;

$\mathrm{TS}+2 \mathrm{O}=$ Tetramer $\left\{4 \mathrm{M} \rightarrow 4 \mathrm{Cu}^{2+}+2 \mathrm{O}^{2-}\right\}$ Square;

$\mathrm{TZ}+2 \mathrm{O}=$ Tetramer $\left\{4 \mathrm{M} \rightarrow 4 \mathrm{Cu}^{2+}+2 \mathrm{O}^{2-}\right\}$ Zigzag;

$\mathrm{H}+4 \mathrm{O}=$ Hexamer $\left\{6 \mathrm{M} \rightarrow 6 \mathrm{Cu}^{2+}+4 \mathrm{O}^{2}-\right\}$;

$\mathrm{DS}+4 \mathrm{O}=$ Double Square $\left\{7 \mathrm{M} \rightarrow 7 \mathrm{Cu}^{2+}+4 \mathrm{O}^{2-}\right\}$.

Besides, $\mathrm{Zn}^{2+}$ clusters will squeeze out oxygen ion, which is marked as $\mathrm{TL}-\mathrm{O}$ and so on. «-O» represents an $\mathrm{O}^{2-}$ ion is squeeze out the cluster. 


\subsection{X-ray diffraction results and cuprate superconductivity}

XRD results show that undoped and low-doped Y-123 samples have the well single phase. As doped concentration $x$ increase, samples show slight impure phases at $x=$ $=0.12, x=0.20$ and at $x=0.25$ for $\mathrm{Zn}, \mathrm{Al}$, and Co doping, respectively. Here in-order three $x$ values correspond with the average binding energy $E_{m}$ of $\mathrm{Zn}, \mathrm{Al}$, and Co. Every cluster in Table 1, namely, $x(\mathrm{Zn})<x(\mathrm{Al})<x(\mathrm{Co})$ has the same relation to $E_{m}(\mathrm{Zn})<E_{m}(\mathrm{Al})<E_{m}(\mathrm{Co})$. Such a result reveals that the small theoretic $E_{m}$ samples form easily the impure phases in the experiments, so our theoretic calculations are able to reflect the experimental data. All the lattice parameters were calculated by the least square method with powder XRD data, and the results of $\mathrm{Zn}$ and $\mathrm{Al}$ doped samples are shown in Fig. 1 and Fig. 2. Due to Co doped samples have the similar results to Al doped, the former lattice parameters are not illustrated. For $\mathrm{Zn}$ doped samples, the lattice parameter $a$ and $b$ increase slightly with $x$ increasing, because the $\mathrm{Zn}^{2+}$ radius $(0.74 \AA)$ is larger than the $\mathrm{Cu}^{2+}$ radius $(0.72 \AA)$, but the $\mathrm{O}-\mathrm{T}$ transition does not appear in $x=0-0.4$. While for $\mathrm{Al}$ and Co doping, $\mathrm{O}-\mathrm{T}$ transition occurs near $x=0.15$ and 0.12 , respectively.

Generally, $\mathrm{O}-\mathrm{T}$ transition results from the change of oxygen content in $\mathrm{Cu}-\mathrm{O}$ chains [20-23]. Thus the variation of lattice parameters $a$ and $b$ indicates that $\mathrm{Al}$ and Co ions enter mainly $\mathrm{Cu}(1)$ sites, which is consistent with Hoffmann et al. experiments [37,38]. It should be noticed that $\mathrm{O}-\mathrm{T}$ transition occurs before the appearance of impure phases, which reflects the intrinsic properties of $\mathrm{Al}$ and $\mathrm{Co}$ doped Y123 systems. For the same reason, the variation of lattice parameters shows that $\mathrm{Zn}$ ions enter mainly $\mathrm{Cu}(2)$ sites on $\mathrm{CuO}_{2}$ planes $[39,41]$, where the superconductivity occurs. It implies that the superconductivity suppressed by $\mathrm{Zn}$ doping should be stronger than by $\mathrm{Al}$ and Co doping. In our experiments [20,22-24], the $T_{c}$ is $92 \mathrm{~K}$ for Y123 compounds without elemental substitution, which call undoped

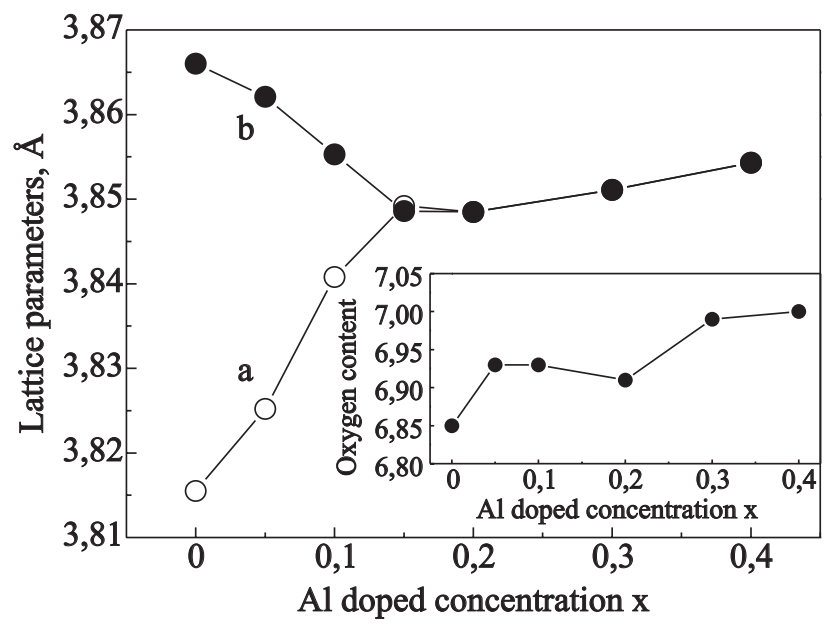

Fig. 1. Lattice parameter $a$ and $b$ variation with $\mathrm{Al}$ doped concentration $x$ in YBCO systems. The insert picture indicates the oxygen content variation with $x$ (Ref. 17).

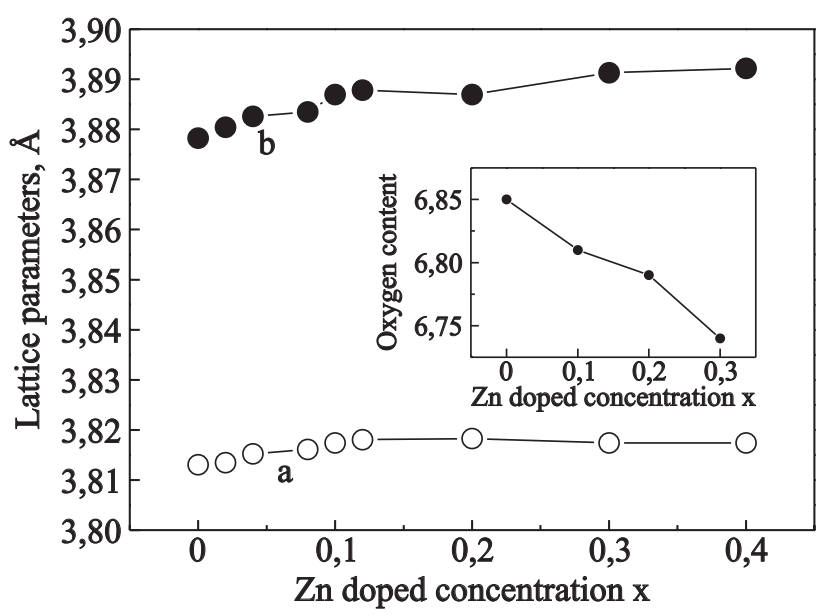

Fig. 2. Lattice parameter $a$ and $b$ variation with $\mathrm{Zn}$ doped concentration $x$ in YBCO systems. The insert picture indicates the oxygen content variation with $x$ (Ref. 17).

samples in the text. For Al doping, during $x=0-0.10$, the $T_{c}$ falls slightly to $91 \mathrm{~K}$; at $x=0.20$, the $T_{c}$ is $83.2 \mathrm{~K}$; and at $x=0.40, T_{c}=42.5 \mathrm{~K}$. And the $T_{c}$ falls to about $72.3 \mathrm{~K}$ for $\mathrm{Zn}$ concentration $x=0.10$; when $x=0.20$, the $T_{c}$ is $42.6 \mathrm{~K}$ and for $x=0.40, T_{c}=15.7 \mathrm{~K}$. While for Co doped samples, the $T_{c}$ decreases stepwise with $x$ increasing, when $x=0.05$, the $T_{c}$ is about $80.0 \mathrm{~K}$; at $x=0.10$, the $T_{c}$ is $67.5 \mathrm{~K}$; at $x=0.20$, the $T_{c}$ is $44.5 \mathrm{~K}$; and if $x=0.30$, one has $T_{c}=21.0 \mathrm{~K}$. Obviously, when $x<0.20$, Zn doping suppresses the superconductivity much more severely than Al and Co doping, however, when $x>0.20$, Co doping suppresses most strongly the superconductivity in the three doped ions. It suggests that the cluster effect is still an important factor in suppression of the superconductivity because Co ions show the strongest cluster effect as above calculation results.

\subsection{Oxygen content variations}

The insert picture in Fig. 1 and Fig. 2 show the experimental results of oxygen content concerning $\mathrm{Al}$ and $\mathrm{Zn}$ doped samples [17]. Here the oxygen content of Co doping is omitted as being similar to $\mathrm{Al}$ doping. For $\mathrm{M}^{3+}\left(\mathrm{Al}^{3+}\right.$, $\mathrm{Co}^{3+}$ ) doping, $\mathrm{M}^{3+}$ valence is higher than $\mathrm{Cu}^{2+}$ in $\mathrm{Cu}-\mathrm{O}$ chains, the dispersed ions cannot capture one oxygen ion due to charge equilibrium. As $x$ increases the ions form clusters, which are able to capture oxygen ions, so the oxygen content should rise in $\mathrm{Al}$ and Co doped samples. However, for $\mathrm{Zn}$ doping, the oxygen content falls obviously with $x$ increase, such variation characteristics are consistent with the simulated calculations, because $\mathrm{Zn}^{2+}$ valence is lower than $\mathrm{Cu}^{2.25+}$ and the dispersed ions cannot extrude oxygen ions to achieve electric neutrality. While the cluster has not formed yet and $\mathrm{Zn}$ ions are distributed randomly, there are hardly oxygen vacancies around $\mathrm{Zn}$ ions and the oxygen content should not decrease. With $x$ increasing, $\mathrm{Zn}$ ions form more and more clusters, because $\mathrm{Zn}$ ions require less oxygen ions for coordination than $\mathrm{Cu}$, 
the extra oxygen ions will be extruded from the clusters. Therefore, oxygen vacancies would appear on $\mathrm{CuO}_{2}$ planes, and then the oxygen content should descend noticeably (Fig. 2, insert). We can conclude that cluster effect is indeed a factor that causes oxygen content to reduce in $\mathrm{Zn}$ doped YBCO. From the above analysis, oxygen content variation characteristics in those doped samples can be explained convincingly by the cluster effect. In fact, the PAT experiments have the same conclusions.

\subsection{Positron experiments}

PAT experiments are described in detail in our other papers [20-24]. The positron lifetime is defined as the inverse of annihilation rate. According to the model of twostate capture in condensed matter [42] with the short lifetime $\tau_{1}$ and the long lifetime $\tau_{2}$, the processes of positron annihilation are attributed to free-state annihilation and trapping-state annihilation, respectively. The former intensity $I_{1}$ denotes the proportion of free-state annihilation to total annihilation events, the latter intensity $I_{2}=1-I_{1} . \tau_{1}$ reflects mainly the annihilation process in the perfect crystal lattices and can be used to detect the electron distribution of the inner microstructure. $\tau_{2}$ reflects mainly the process of positron captured in the imperfect areas inside the materials; it can characterize the intrinsic structure and preparation quality of samples. If positrons annihilate in the imperfections, such as oxygen vacancy, twin boundary, dislocation, and cation vacancies, $\tau_{2}$ will be larger, sometimes it is noted as $\tau_{3}$, generally $\tau_{3}>800$ ps [43-45]. Our samples do not contain $\tau_{3}$ component, this indicates the credibility of the sample quality.

According to the characteristics of positron annihilation, local electron density $n_{e}=1 /\left(\pi r_{0}^{2} c \tau_{\text {bulk }}\right)$, where $r_{0}$ is the classical electron radius, $c$ is the velocity of light, and $\tau_{\text {bulk }}$ is the systematical lifetime parameter, it is defined as:

$$
1 / \tau_{\text {bulk }}=I_{1} / \tau_{1}+I_{2} / \tau_{2}
$$

Some relational calculation results $[46,47]$ show that about $90 \%$ positrons annihilate with the valence electrons, and only about $10 \%$ positrons annihilate with the electrons within the atomic kernel. These results indicate that the $n_{\boldsymbol{e}}$ amount is determined mainly by the valence electrons, so we may regard the density of valence electron as the $n_{\boldsymbol{e}}$ without influence on the general conclusions.

When $\mathrm{M}^{+3}\left(\mathrm{Al}^{+3}, \mathrm{Co}^{+3}\right)$ enter $\mathrm{Cu}-\mathrm{O}$ chains as above, the dispersive $\mathrm{M}^{+3}$ contains one valence electron less than $\mathrm{Cu}^{2+}$, so the $n_{e}$ should drop. However, the doped ions form clusters as $x$ increases, oxygen ions will be attracted into the crystal lattice. Once introduced by the clusters, every oxygen ion will attract two valence electrons in terms of charge equilibrium. Therefore the valence electron density rises, namely, the $n_{e}$ shows a rising trend. When the $n_{e}$ dropping and rising reach a balance, the low saturation emerges as shown in the experiments [24]. Obviously, such a result originates from the cluster effect. In contrast, while +2 valence ions enter $\mathrm{CuO}_{2}$ planes, $\mathrm{Zn}^{2+}$ loses fewer electrons than $\mathrm{Cu}^{2.25+}$, so the density should rise on the consideration of valence state. However, as $x$ increase, the $\mathrm{Zn}$ ions start to form clusters. As mentioned before, every four $\mathrm{Zn}^{2+}$ will extrude an $\mathrm{O}^{-}$(including a hole) which carries away two valance electrons, so the density begin to fall. When the rising and falling of the density reach a balance, the density $n_{e}$ tends to high saturation [20], which is influenced evidently by the cluster effect. As a result, the $n_{e}$ saturation also can be elucidated satisfactorily through the cluster effect as the oxygen content variations above.

\subsection{Valence electron density and the $T_{c}$ of cuprates}

Figure 3 shows the relationship between the $T_{c}$ and the reduced $n_{e}$ in $\mathrm{Al}, \mathrm{Zn}$ and Co doped samples. For $\mathrm{Al}$ and Co doped samples, both reduced $n_{e}$ have the great decrease in the little doping, the $T_{c}$ descends only slightly, especially in the Al doped samples, but the reduced $n_{e}$ of Co doped samples descends faster than that of $\mathrm{Al}$ doped in the same concentration $x$, as shown in the figure, so the $n_{e}-T_{c}$ curve turning point in Co doped samples is further from $n_{e}=1.00$ than that in Al doped, such a result still gives evidence that the $E_{m}$ is larger in Co doped samples. Below the curve turning point in both samples, the $T_{c}$ seems to lose further the association with the reduced density $n_{e}$, in particular, when both $T_{c}$ drop sharply, the reduced $n_{e}$ varies only slightly. In fact, when $x$ is low, both ions enter the crystal lattice in individual, $\mathrm{M}^{3+}$ loses more valence electrons than $\mathrm{Cu}^{2+}$, so the density $n_{e}$ will decrease. However, the $T_{c}$ descends slightly with the density change because the dispersed ions cannot destroy the crystal lattice. Below the curve turning point the $T_{c}$ starts to drop sharply with clusters appearing. On our idea, the stronger cluster effect is, the stronger it will distort the structure of crystal lattice. So the superconductivity is seriously suppressed in both samples. In contrast with both above, the $n_{e}-T_{c}$ curve of $\mathrm{Zn}$ doping has the opposite variation trend in the beginning stage, though the reduced density raises greatly, the $T_{c}$ descends still slightly, below the curve turning point the $T_{c}$

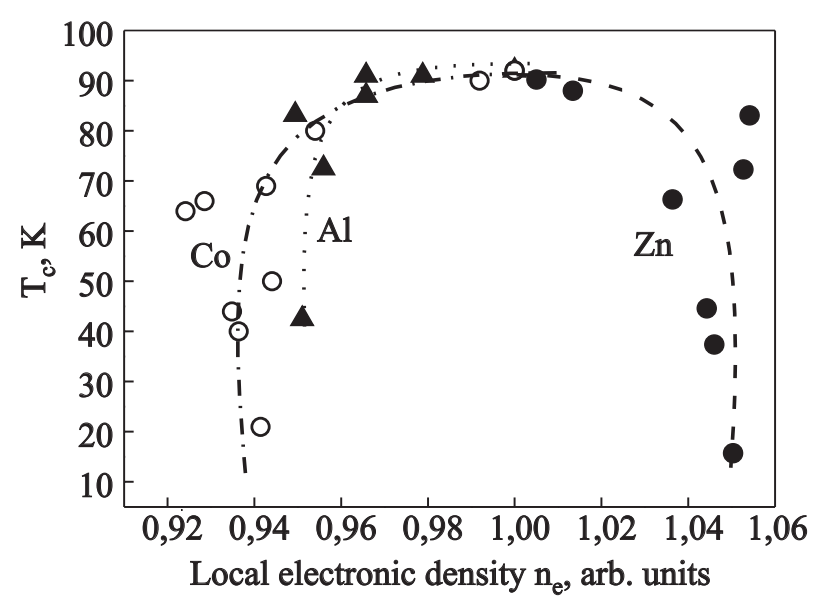

Fig. 3. The variation of superconducting transition temperature $T_{c}$ with reduced valence electron density $n_{e}$ in $\mathrm{Al}, \mathrm{Zn}$ and Co doped YBCO systems. 
starts to drop sharply from $72.3 \mathrm{~K}$ to $15.7 \mathrm{~K}$, whereas the reduced $n_{e}$ increases only slightly. As above statements, similarly, when $x$ is small, $\mathrm{Zn}$ ions enter the crystal lattice in individual; $\mathrm{Zn}^{2+}$ loses fewer electrons than $\mathrm{Cu}^{2.25+}$, so the density will increase. However, the $T_{c}$ descends slightly with the density increasing, because the dispersed ions do not destroy the crystal lattice. While the $\mathrm{Zn}$ clusters appear in the doped samples, the structures of crystal lattice will be distorted by the cluster effect, which will influence directly the pairing and transportation of carriers, and thus the superconductivity is suppressed markedly, then the $T_{c}$ starts to drop sharply. Anyway, when the reduced density increases or decreases essentially, the $T_{c}$ alters only slightly; on a contrary, while the $T_{c}$ falls dramatically, the reduced density changes hardly. As a result, we can conclude that the $T_{c}$ has no direct connection with the valence electron density. However, when the cluster effect strengthens in the region of turning point, the $T_{c}$ falls noticeably. Therefore, the cluster effect is an important factor that suppresses the cuprate superconductivity.

\section{Conclusions}

In conclusion, the high- $T_{c}$ cuprates $\mathrm{YBa}_{2} \mathrm{Cu}_{3-x}(\mathrm{Al}, \mathrm{Zn}, \mathrm{Co})_{x} \mathrm{O}_{7-\delta}$ $(x=0-0.5)$ have been analyzed and studied systematically by XRD, positron annihilation technique, oxygen content, and calculations of binding energy. The simulated calculations, the variations of oxygen content and positron experiments support congruously the existence of cluster effect. Especially, it is reasoned that the cluster effect is an important factor in suppression of high- $T_{c}$ cuprate superconductivity and the $T_{c}$ has no direct connection with the density of valence electron.

This work is supported by The Natural Science Foundation of China (No.10647145).

1. U. Schwingenschlogl and C. Schuster, Phys. Rev. B79, 092505 (2009).

2. H. Yamase, Phys. Rev. B79, 052501 (2009).

3. X. Wang, A. Dibos, and J.Z. Wu, Phys. Rev. B77, 144525 (2008).

4. H.H. Song, M.W. Davidson, and J. Schwartz, Supercond. Sci. Tech. 22, 062001 (2009).

5. W. Markowitsch, W. Lang, J.D. Pedarnig, and D. Bauerle, Supercond. Sci. Tech. 22, 034011 (2009).

6. J. Zaanens and T. Senthil, Nature Phys. 2,138 (2006).

7. J. Guo, C. Dong, H. Gao, H.H. Wen, L.H. Yang, F. Zeng, and H. Chen, Chin. Phys. B17, 1124 (2008).

8. C. Dong, Chin. Phys. B15, 3005 (2006).

9. L.B. Shi, Y. Zheng, J.Y. Ren, M.B. Li, and G.H. Zhang, Acta Phys. Sin. 57, 1183 (2008).

10. Y. Fukuuzumi, K. Mizuhaohi, K. Takenaka, and S. Uchida, Phys. Rev. Lett. 76, 684 (1996).

11. H. Salamati and M. Mohammadi, J. Phys. IV France 123, 19 (2005).
12. B.Nachumi, A. Keren, K. Kojima, M. Larkin, G.M. Luke, O. Tcheryshov, Y.J. Uemura, N. Ichikawa, M. Goto, and S. Uchida, Phys. Rev. Lett. 77, 5421 (1996).

13. Y.K. Kuo, C.W. Schneider, M.J. Skove, M.V. Nevitt, G.X. Tessema, and J.J. Mcgee, Phys. Rev. B56, 6201 (1997).

14. H. Takagi, Physica C341-348, 3 (2000).

15. A.H. Macdonald, Nature 414, 409 (2001).

16. M. Capone, M. Fabrizio, C. Castellani, and E. Tosatti, Science 296, 2364 (2002).

17. M. Tarascon, P. Barboux, P.F. Miceli, L.H. Greene, G.W. Hull, M. Eibschutz, and S.A. Sunshine, Phys. Rev. B37, 7458 (1988).

18. R.S. Horland and T.H. Geballe, Phys. Rev. B39, 9017 (1989).

19. J. Axnäs, W. Holm, Yu. Eltsev, and Ö. Rapp, Phys. Rev. B53, 3003 (1996).

20. P.L. Li, J.C. Zhang, G.X. Cao, C. Jing, and S.X. Cao, Phys. Rev. B69, 224517 (2004).

21. P.L. Li, J.C. Zhang, G.X. Cao, D.M. Deng, L.H. Liu, C. Dong, C. Jing, and S.X. Cao, Acta Phys. Sin. 53, 1223 (2004).

22. A.H. Wang, X.X. Wang, S.F. Li, J. Zhang, H.Q. Lu, L.M. Gao, X.L. Li, Y.Y. Wang, and P.L. Li, J. Low Temp. Phys. 149, 89 (2007).

23. P.L. Li, Y.Y. Wang, Y.T. Tian, J. Wang, X.L. Niu, J.X. Wang, D.D. Wang, and X.X. Wang, Chin. Phys. B17, 3483 (2008).

24. A.H. Wang, X.X. Wang, Y.G. Cao, X.L. Li, Y.Y. Wang, L.M. Gao, H.Q. Lu, J. Zhang, and P.L. Li, Fiz. Nizk. Temp. 34, 219 (2008) [Low Temp. Phys. 34, 168 (2008)].

25. R.P. Gupta and M. Gupta, Physica C305, 179 (1998).

26. M.S. Islam and C. Aanathamohan, Phys. Rev. B44, 9492 (1991).

27. K. Saarinen, J. Niddils, H. Kauppinen, M. Hakala, M.J. Puska, P. Hautojarvi, and C. Corbel, Phys. Rev. Lett. 82, 1883 (1999).

28. A.I. Kul'menrt'ev, Eur. Phys. J. Appl. Phys. 25, 191 (2004).

29. T.E. M. Staab, M. Haugk, Th. Frauenheim, and H.S. Leipner, Phys. Rev. Lett. 83, 5519 (1999).

30. A. Somoza, A. Dupasquier, I.J. Polmear, P. Folegati, and R. Ferragut, Phys. Rev. B61, 14454 (2000).

31. T. Banerjee, R.N. Viswanath, D. Kanjilal, R. Kumar, and S. Ramasamy, Solid State Commun. 114, 655 (2000).

32. D. Udayan, D. Sanyal, S. Chauahuri, P.M. G. Nambissan, T. Wolf, and H. Wuhl, Phys. Rev. B62, 14519 (2000).

33. Y.C. Jean, J. Kyle, H. Nakanishi, and P.E.A. Turchi, Phys. Rev. Lett. 60, 1069 (1988).

34. J.C. Zhang, F.Q. Liu, and G.S. Cheng, Phys. Rev. A201, 70 (1995).

35. J.C. Zhang, L.H. Liu, C. Dong, J.Q. Li, H. Chen, X.G. Li, and G.S. Cheng, Phys. Rev. B65, 054513 (2002).

36. P.C. Li, H.S. Yang, Z.Q. Li, Y.S. Chai, and L.Z. Cao, Chin. Phys. 11, 285 (2002).

37. J.F. Bringley, T.M. Chen, B.A. Averill, K.M. Wong, and S.J. Poon, Phys. Rev. B38, 2432 (1988).

38. L. Hoffmann, A.A. Manuel, M. Peter, E. Walker, M. Gauthier, A. Shukla, B. Barbiellini, S. Massidda, Gh. Adam, 
W.N. Hardy, and R.X. Liang, Phys. Rev. Lett. 71, 4047 (1993).

39. J.M. Tarascon, L.H. Greene, P. Barboux, W.R. McKinnon, G.W. Hull, T.P. Orlando, K.A. Delin, S. Foner, and E.J. McNiff, Jr., Phys. Rev. B36, 8393 (1987).

40. G. Xiao, M.Z. Cieplak, and C.L. Chien, Phys. Rev. B42, 240 (1990).

41. N. Bulut, D. Hone, D.J. Scalapino, and E.Y. Loh, Phys. Rev. Lett. 62, 2192 (1989).

42. P. Haotuojiawei, Positron-Annihilation Technology, Science Press (1983), p. 255.
43. C. Nagel, K. Ratzke, E. Schmidtke, F. Faupel, and W. Ulfert, Phys. Rev. B60, 9212 (1999).

44. A. Somoza, A. Dupasquier, I.J. Polmear, P. Folegati, and R. Ferragut, Phys. Rev. B61, 14454 (2000).

45. L.J. Li, Z.X. Wang, and J.L. Wu, Acta Phys. Sin. 47, 844 (1998).

46. K.O. Jensen, R.M. Nieminen, and M.J. Puaka, J. Phys. Cond. Matter 1, 3727 (1989).

47. H.B. Zhang and H. Sato, Phys. Rev. Lett. 70, 1697 (1993). 